\title{
Asymptotic Likelihood-Based Prediction Functions
}

\author{
Thomas F. Cooley; William R. Parke
}

Econometrica, Vol. 58, No. 5. (Sep., 1990), pp. 1215-1234.

Stable URL:

http://links.jstor.org/sici?sici=0012-9682\%28199009\%2958\%3A5\%3C1215\%3AALPF\%3E2.0.CO\%3B2-T

Econometrica is currently published by The Econometric Society.

Your use of the JSTOR archive indicates your acceptance of JSTOR's Terms and Conditions of Use, available at

http://www.jstor.org/about/terms.html. JSTOR's Terms and Conditions of Use provides, in part, that unless you have obtained prior permission, you may not download an entire issue of a journal or multiple copies of articles, and you may use content in the JSTOR archive only for your personal, non-commercial use.

Please contact the publisher regarding any further use of this work. Publisher contact information may be obtained at http://www.jstor.org/journals/econosoc.html.

Each copy of any part of a JSTOR transmission must contain the same copyright notice that appears on the screen or printed page of such transmission.

JSTOR is an independent not-for-profit organization dedicated to and preserving a digital archive of scholarly journals. For more information regarding JSTOR, please contact support@jstor.org. 


\title{
ASYMPTOTIC LIKELIHOOD-BASED PREDICTION FUNCTIONS
}

\author{
By Thomas F. Cooley and William R. Parke ${ }^{1}$
}

\begin{abstract}
This paper develops asymptotic prediction functions that approximate the shape of the density of future observations and correct for parameter uncertainty. The functions are based on extensions to a definition of predictive likelihood originally suggested by Lauritzen and Hinkley. The prediction function is shown to possess efficiency properties based on the Kullback-Leibler measure of information loss. Examples of the application of the prediction function and the derivation of relative efficiency are shown for linearnormal models, nonnormal models, and ARCH models.
\end{abstract}

KEYwords: Prediction, predictive likelihood, predictive efficiency, nonnormal errors, ARCH models.

\section{INTRODUCTION}

Although PREDICTION is often a primary goal of econometric research, problems of predictive inference have received relatively little attention in the literature. A glance at any econometrics text reveals only a few pages devoted to problems of prediction, the major concern being with problems of parametric estimation and inference. This neglect may stem from the fact that no one frequentist technique is accepted as universally appropriate for predictive inference. In practice the prediction problem is approached by a diverse collection of techniques whose properties are not always well understood. Recent papers by Fair (1980) and Brown and Mariano (1983, 1984, 1985) have furthered understanding of some common procedures for generating predictions, but a unified basis for evaluating them is still missing. The Bayesian viewpoint provides a consistent theory of prediction but implementation in complex problems is often difficult. Our objective in this paper is to suggest a class of likelihood based prediction functions that is widely applicable. The likelihood concept proposed has the advantage that it puts predictive inference on a consistent footing, a role similar to that played by the likelihood principle of estimation. The use of a formal definition of predictive likelihood also provides a reference point for the interpretation of existing approaches to prediction.

The properties of commonly used prediction methods have been studied in a number of papers that try to rationalize their performance in the context of models with well defined characteristics. Bianchi \& Calzolari (1980) and Fair (1980) among others have studied the behavior of Monte Carlo predictors of various sorts to ascertain the contribution of different sources of uncertainty to prediction error. In a series of papers Mariano and Brown have compared the asymptotic properties of deterministic predictors, which replace structural dis-

\footnotetext{
${ }^{1}$ We have received helpful comments from Sean Collins, Mark Feldman, and two anonymous referes. We are especially grateful to a co-editor who made many insightful suggestions. The responsibility for errors is our own.
} 
turbances by their expected values, with stochastic predictors based on drawings of the disturbances. The latter include straightforward Monte Carlo predictors as well as stochastic predictors based on the use of sample period residuals.

The approach taken in the current paper is in the same spirit as the research just cited. We emphasize accounting for the uncertainty due to stochastic disturbances and, particularly, the uncertainty due to the use of estimated parameters. In contrast to earlier research, however, we emphasize obtaining analytic prediction functions that approximate the entire distribution of future observations rather than focusing on the bias properties of alternative point predictors. We do this because many interesting econometric prediction problems are characterized by predictive distributions that are nonnormal, and, hence, not well characterized by the mean and variance alone. Prediction functions that approximate well the distribution of future observations will be important for obtaining accurate confidence intervals or probability statements about predictions.

The basis for our approach to prediction functions is a definition of likelihood due originally to Lauritzen (1974) and Hinkley (1979). Their definition has been applied by Butler (1986) and Cooley, Parke, and Chib (1989). In this paper we extend the Lauritzen-Hinkley definition in a way that permits direct application to more complex econometric problems. We also introduce the concepts of predictive consistency and first and second order predictive efficiency. These are shown to be necessary to discriminate among alternative prediction functions.

In the next section we review the prediction problem and the most commonly used predictors. The Lauritzen-Hinkley definition of predictive likelihood is presented. Our asymptotic likelihood prediction function is presented in Section 3 and the relationship to mean-squared error prediction functions is discussed. We introduce definitions of predictive consistency and efficiency based on the Kullback-Leibler information measure in Section 4. Section 5 extends the definition to cover the use of consistent, but possibly inefficient parameter estimates. Finally, in Section 6, the usefulness of our prediction function is illustrated in the context of regression models with nonnormal disturbances and autoregressive conditional heteroskedasticity $(\mathrm{ARCH})$ models.

\section{PREDICTION FUNCTIONS}

Suppose interest centers on predictions of a random variable $y_{i}$ defined over the space $Y$. The $m$ data period observations $\left(y_{t}: t=1, \ldots, m\right)$ are denoted by $y_{d}$. The $n$ future period observations that we wish to predict, $\left(y_{t}: t=\right.$ $m+1, \ldots, m+n)$, are denoted by $y_{f}$. The most informative possible statement about the future is the density $f\left(y_{f} \mid \theta\right)$, where $\theta$ is a vector of true parameters contained in a parameter space $\Theta$. Knowledge of $f\left(y_{f} \mid \theta\right)$ permits one to make a variety of point forecasts (mean, median, or mode) and to construct confidence regions for predictions. Because $\theta$ is unknown, practical prediction procedures most often generate point estimates of $y_{f}$ based on point estimates of $\theta$ and in some cases attempt to estimate the second moment of $y_{f}$. 
We suppose that the model generating realizations of $y_{f}$ can be represented as

$$
y_{f}=g\left(x_{f}, u_{f}, \theta\right),
$$

where $x_{f}$ is a vector of exogenous variables and $u_{f}$ is a vector of stochastic disturbances. ${ }^{2}$ A predictor is defined by making specific assumptions about $u_{f}$ and $\theta$. Mariano and Brown define the deterministic predictor based on a consistent estimate $\hat{\theta}_{d}$ of $\theta$ to be

$$
y_{f}=g\left(x_{f}, 0, \hat{\theta}_{d}\right),
$$

where the error term is set equal to its expected value. An alternative to the deterministic predictor is the Monte Carlo predictor defined as

$$
\boldsymbol{y}_{f}=g\left(x_{f}, \boldsymbol{u}_{f}, \hat{\boldsymbol{\theta}}_{d}\right) \text {, }
$$

where the $\boldsymbol{u}_{f}$ represent draws from some specified distribution of $u_{f}$ and $\boldsymbol{y}_{f}$ represents the corresponding set of realizations of $y_{f}$. A second form of Monte Carlo predictor that is often used (Muench et al. (1974), Fair (1980)) is defined by draws of both error terms and coefficients

$$
\boldsymbol{y}_{f}=g\left(x_{f}, \boldsymbol{u}_{f}, \hat{\boldsymbol{\theta}}_{d}\right),
$$

where here $\hat{\boldsymbol{\theta}}_{d}$ denotes drawings from the asymptotic distribution of the estimated coefficients.

Although interest typically focuses on the first and second moments of the distributions generated by (2.1) and (2.2), the entire distribution is of interest as an approximation to $f\left(y_{f} \mid \theta\right)$. Indeed, the Monte Carlo procedure described by (2.1) can be thought of as an attempt to capture the density ${ }^{3}$

$$
f\left(y_{f} \mid \hat{\theta}_{d}\right)
$$

by drawing the error terms. The second Monte Carlo procedure attempts to weight the density (2.3) by drawings from the asymptotic distribution of the $\hat{\theta}_{d}$ 's:

$$
\int f\left(y_{f} \mid \hat{\boldsymbol{\theta}}_{d}\right) e^{-1 / 2\left(\hat{\boldsymbol{\theta}}_{d}-\hat{\boldsymbol{\theta}}_{d}\right)^{2} / V\left(\hat{\boldsymbol{\theta}}_{d}\right)} d \hat{\boldsymbol{\theta}}_{d}
$$

The obvious drawback to (2.1) is that it ignores the uncertainty introduced by using $\hat{\theta}_{d}$, while (2.2) appears to take account of it, but does so in a way that is

\footnotetext{
${ }^{2}$ Uncertain future exogenous variables and dependent observations both raise issues not covered by this discussion. To focus attention on the problem of predicting $y_{f}$, we assume that the exogenous variables $x_{f}$ are perfectly predictable, and for notational simplicity we will subsume $x_{f}$ into the notation $f\left(y_{f} \mid \theta\right)$. Dependent observations could be handled in principle by explicitly recognizing the conditioning $f\left(y_{f} \mid y_{d}, \theta\right)$ although Phillips (1979) points out that the distributional dependence of the relevant terminal observations in $y_{d}$ and the data period parameter estimates $\hat{\theta}_{d}$ may not be analytically trivial. We address this last issue in the context of the ARCH model in Section 6.

${ }^{3}$ In order to preserve a consistent notation throughout we denote densities where parameter estimates have been substituted for true values as conditional densities $f(\cdot \mid \cdot)$ while recognizing that this constitutes a slight abuse of notation.
} 
difficult to judge without reference to some theoretical standard. The procedures developed in this paper have a lot in common with Monte Carlo methods. They involve corrections to forecasting densities to account for parameter uncertainty and will typically be implemented by simulation, but they are motivated theoretically in the following sections.

An alternative to the approaches just discussed is to eliminate the unknown parameters $\theta$ by the use of sufficient statistics. This is the basis of the notion of predictive likelihood that was originally suggested by Lauritzen (1974) and Hinkley (1979). The Lauritzen-Hinkley concept recognizes the central importance of $f\left(y_{f} \mid \theta\right)$ for problems of prediction, but uses sufficient statistics to eliminate the unknown parameter $\theta$. Let $S_{d}, S_{f}$, and $S_{d+f}$ be sufficient reductions of $Y_{d}, Y_{f}$, and their union respectively. Sufficiency ensures that the density $f\left(y_{d} \mid \theta\right)$ can be factored as

$$
f\left(y_{d} \mid \boldsymbol{\theta}\right)=f\left(y_{d} \mid S_{d}\right) f\left(S_{d} \mid \boldsymbol{\theta}\right),
$$

where $f\left(y_{d} \mid S_{d}\right)$ does not depend on $\theta$. The Lauritzen-Hinkley definition of predictive likelihood exploits the fact that $S_{d+f}$ is a function of $S_{f}$ and $S_{d}$ that does not depend on $\theta$.

DefinItION 1 (Lauritzen-Hinkley): The predictive likelihood function is

$$
\operatorname{plik}\left(y_{f} \mid y_{d}\right)=f\left(y_{f}, S_{d} \mid S_{d+f}\right)=\frac{f\left(y_{f} \mid \theta\right) f\left(S_{d} \mid \theta\right)}{f\left(S_{d+f} \mid \theta\right)}
$$

This definition envisions treating $\operatorname{plik}\left(y_{f} \mid y_{d}\right)$ as a likelihood function for the future observations $y_{f}$. In practical applications the plik could be used to order future values by their plausibility and to obtain confidence intervals for $y_{f}$. This definition has been applied to several econometric problems by Cooley, Parke, and Chib (1987), but its applicability is limited. There are some problems for which there is no sufficient reduction of the data-probit models are one example. There are many other examples where minimal sufficient statistics exist but have unworkably complex distributions-logit models are an example. In the next section we develop an alternative definition that is applicable and easily implemented in these situations.

\section{ASYMPTOTIC PREDICTION FUNCTIONS}

The limitations of the preceding definition of predictive likelihood are not insurmountable. First, we know that maximum likelihood estimates are asymptotically sufficient. These provide a solution to problems that do not admit sufficient statistics. Second, we can replace the (often intractable) exact distributions in the Lauritzen-Hinkley definition with asymptotic distributions. In Appendix A we show how to use a series of asymptotically valid approximations to 
arrive at the following definition:

DEFINITION 2: The asymptotic predictive likelihood function is

$$
\operatorname{plik}^{a}\left(y_{f} \mid \hat{\boldsymbol{\theta}}_{d}\right)=f\left(y_{f} \mid \hat{\boldsymbol{\theta}}_{d}\right) \cdot \exp \left\{w_{1}\left(y_{f} ; \hat{\boldsymbol{\theta}}_{d}\right)+w_{2}\left(y_{f} ; \hat{\boldsymbol{\theta}}_{d}\right)\right\},
$$

where

$$
\begin{aligned}
& w_{1}\left(y_{f} ; \hat{\theta}_{d}\right)=-\frac{1}{2} \nabla\left(y_{f} ; \hat{\theta}_{d}\right) H\left(y_{d+f} ; \hat{\theta}_{d}\right)^{-1} \nabla\left(y_{f} ; \hat{\theta}_{d}\right)^{\prime}, \\
& w_{2}\left(y_{f} ; \hat{\theta}_{d}\right)=\nabla\left(y_{f} ; \hat{\theta}_{d}\right) \psi\left(\hat{\theta}_{d}\right)-\frac{1}{2} \operatorname{tr}\left[H\left(y_{f} ; \hat{\theta}_{d}\right) H\left(y_{d} ; \hat{\theta}_{d}\right)^{-1}\right],
\end{aligned}
$$

$\nabla\left(y_{f} ; \hat{\theta}_{d}\right)$ is the $\log$ gradient function of $f\left(y_{f} \mid \theta\right)$ evaluated at $y_{f}$ and $\hat{\theta}_{d}$, $H\left(y_{d+f} ; \hat{\theta}_{d}\right)$ is the log Hessian of $f\left(y_{d+f} \mid \theta\right)$, and $\psi\left(\hat{\theta}_{d}\right)$ is the $O\left(m^{-1}\right)$ bias in the MLE $\hat{\theta}_{d}$.

Despite a bit of notational complexity, (3.1) has a practical form that can be implemented easily for common econometric prediction problems. The first and second derivatives of the log density are usually not difficult to compute, and (3.1) can often be incorporated into a Monte Carlo simulation strategy. This definition applies strictly to models with independent observations.

The elements of (3.1) have the following intuitive justification. The first term on the right-hand side of (3.1) is simply the prediction function that would obtain if we knew the correct functional form of $f\left(y_{f} \mid \theta\right)$, but substituted consistent estimates for the unknown parameters. We will refer to this as the certainty equivalence (CEQ) prediction function (although it should be noted that the term is wishful rather than descriptive as no equivalence exists). It is, as noted in the previous section, the form one is approximating with the Monte Carlo prediction procedures extensively analyzed by Mariano and Brown.

The factor $w_{1}\left(y_{f} ; \hat{\theta}_{d}\right)$ corrects the certainty equivalence prediction function for parameter uncertainty. It typically puts more probability in the tails of a prediction function, where the $\log$ gradient $\nabla\left(y_{f} ; \hat{\theta}_{d}\right)$ is largest. Loosely, this increase in the dispersion of the prediction function relative to the CEQ density recognizes that $y_{f}-\hat{y}_{f}$ will have a greater variance than $y_{f}-E\left(y_{f}\right)$. We formalize this idea in the next section.

The two terms of $w_{2}\left(y_{f} ; \hat{\theta}_{d}\right)$ correct for two related problems. The first adjusts for asymptotic bias of order $O\left(\mathrm{~m}^{-1}\right)$ in the m.l.e., and the second adjusts for the possibility that the second derivative matrix is not constant over $y_{f}$. Both elements could be derived by simply estimating the expectation of a Taylor series approximation to $g\left(y_{f} \mid \theta\right)=\log \left(f\left(y_{f} \mid \theta\right)\right)$ :

$$
\begin{aligned}
g\left(y_{f} \mid \hat{\theta}_{d}\right)-g\left(y_{f} \mid \theta\right)= & \nabla_{f}\left(y_{f} ; \hat{\theta}_{d}\right)\left(\hat{\theta}_{d}-\theta\right) \\
& +\frac{1}{2}\left(\hat{\theta}_{d}-\theta\right)^{\prime} H_{f}\left(y_{f} ; \hat{\theta}_{d}\right)\left(\hat{\theta}_{d}-\theta\right) .
\end{aligned}
$$

The expectation of this is zero for a linear-normal model, but in general it will not be. 
These adjustments for parameter uncertainty can be contrasted with the most commonly used technique for evaluating predictions--mean-squared error (MSE) analysis. An asymptotic MSE analysis is based on a point forecast $\hat{y}_{f}$, which is typically computed by simply setting unknown errors to zero. Because mean-squared error analysis is concerned with only the second moment of $y_{f}-\hat{y}_{f}$, the natural functional form for a prediction function is a normal density

$$
g\left(y_{f} \mid \hat{\theta}_{d}\right) \propto-\frac{1}{2}\left(y_{f}-\hat{y}_{f}\right)^{\prime} V_{f}^{-1}\left(y_{f}-\hat{y}_{f}\right),
$$

where $V_{f}$ is the variance-covariance matrix of $y_{f}$. The usual MSE treatment of parameter uncertainty takes the derivatives $D_{f}=\partial \hat{y}_{f} / \partial \hat{\theta}_{d}$ to be constant even though that generally will not be the case for models with nonlinearities in parameters or dependent observations. Treating $D_{f}$ as constant over $y_{f}$ leads to the approximate first and second derivatives

$$
\begin{aligned}
& \nabla\left(y_{f} ; \hat{\theta}_{d}\right) \approx\left(y_{f}-\hat{y}_{f}\right)^{\prime} V_{f}^{-1} D_{f}, \\
& H_{f} \approx D_{f}^{\prime} V_{f}^{-1} D_{f} .
\end{aligned}
$$

Using (3.3) and (3.4), $w_{1}\left(y_{f} ; \hat{\theta}_{d}\right)$ becomes

$$
w_{1}\left(y_{f} ; \hat{\theta}_{d}\right)=-\frac{1}{2}\left(y_{f}-\hat{y}_{f}\right)^{\prime} V_{f}^{-1} D_{f}\left[H_{d}+D_{f}^{\prime} V_{f}^{-1} D_{f}\right]^{-1} D_{f}^{\prime} V_{f}^{-1}\left(y_{f}-\hat{y}_{f}\right) .
$$

If we ignore any asymptotic bias in $\hat{\theta}_{d}$ and treat $H_{f}$ as constant over $y_{f}$, then the term $w_{2}\left(y_{f} ; \hat{\theta}_{d}\right)$ in (3.1) is constant over $y_{f}$. We can combine (3.2) and (3.5) using the identity (Rao (1973, p. 33))

$$
-V_{f}^{-1}-V_{f}^{-1} D_{f}\left[H_{d}+D_{f}^{\prime} V_{f}^{-1} D_{f}\right]^{-1} D_{f}^{\prime} V_{f}^{-1}=-\left[V_{f}-D_{f}^{\prime} H_{d}^{-1} D_{f}\right]^{-1}
$$

to form the mean squared error prediction function

$$
\operatorname{MSE}\left(y_{f} \mid \hat{\theta}_{d}\right) \propto \exp \left\{-\frac{1}{2}\left(y_{f}-\hat{y}_{f}\right)^{\prime}\left[V_{f}-D_{f} H_{d}^{-1} D_{f}^{\prime}\right]^{-1}\left(y_{f}-\hat{y}_{f}\right)\right\},
$$

which incorporates the variance $V_{f}$ of $y_{f}$ and an approximate variance $-D_{f} H_{d}^{-1} D_{f}^{\prime}$ due to parameter uncertainty, but fails to acknowledge both any nonnormality of $f\left(y_{f} \mid \theta\right)$ and any nonlinearity in the parameter uncertainty. This derivation emphasizes that (3.7) can be regarded as in the same family as $\operatorname{plik}^{a}\left(y_{f} \mid \hat{\theta}_{d}\right)$, but subject to additional linearization.

\section{PREDICTIVE EFFICIENCY}

Having proposed a candidate prediction function we now discuss how to evaluate it. Most common methods of evaluating forecasting errors (e.g. looking at mean-squared errors) are based on the first two moments. This can only make sense to the extent that predictive densities are well approximated by normal distributions. The nonnormal distribution of the forecast errors for many econometric models motivates us to adopt a measure of predictive efficiency that is sensitive to the shape of the future density as well as its 
moments. That measure, the Kullback-Leibler information measure (Kullback (1959)), provides a natural metric for evaluating candidate prediction functions.

In this section, we formalize the information measure of predictive efficiency and then establish four results. First, we derive the information efficiency for the CEQ technique. Second, we establish the order of the relative efficiency gain that can be secured by adjusting the functional form to account for parameter uncertainty. Third, we construct an expansion useful for calculating the efficiency measure for particular prediction functions. Fourth, we show that the predictive likelihood approach yields unambiguous efficiency gains for an important class of location parameter models.

The Kullback-Leibler measure for a particular realized prediction function $f^{*}\left(y_{f} ; \hat{\theta}_{d}\right)$ can be written as:

$$
I\left(f, f^{*}\right)=\int\left[g\left(y_{f} \mid \theta\right)-g^{*}\left(y_{f} \mid \hat{\theta}_{d}\right)\right] f\left(y_{f} \mid \theta\right) d y_{f},
$$

where $g\left(y_{f} \mid \theta\right)=\log \left(f\left(y_{f} \mid \theta\right)\right)$ and $g^{*}\left(y_{f} \mid \hat{\theta}_{d}\right)=\log \left(f^{*}\left(y_{f} \mid \hat{\theta}_{d}\right)\right)$. To abstract from the dependence of (4.1) on the particular realizations of $y_{d}$ and $\hat{\theta}_{d}$, we will compute the expected information loss due to parameter uncertainty

$$
\bar{I}\left(f, f^{*}\right)=\int I\left(f, f^{*}\right) f\left(\hat{\theta}_{d} \mid \theta\right) d \hat{\theta}_{d},
$$

where $f\left(\hat{\theta}_{d} \mid \theta\right)$ is the density of $\hat{\theta}_{d}$.

The asymptotic properties of $\bar{I}\left(f, f^{*}\right)$ will prove both workable and interesting even though evaluating $\bar{I}\left(f, f^{*}\right)$ itself may prove difficult for many typical econometric applications. ${ }^{4}$ Predictive consistency will be defined as ${ }^{5}$

$$
\bar{I}\left(f, f^{*}\right) \rightarrow 0 \quad \text { as } \quad m \rightarrow \infty .
$$

This requires basically that $\hat{\theta}_{d}$ be consistent and that $f^{*}\left(y_{f} \mid \hat{\theta}_{d}\right)$ converge to $f\left(y_{f} \mid \theta\right)$ as $\hat{\theta}_{d} \stackrel{p}{\rightarrow} \theta$.

While a variety of procedures, including CEQ and $\operatorname{plik}^{a}\left(y_{f} \mid y_{d}\right)$, are predictive consistent, it is straightforward to demonstrate that, for nonnormal or nonlinear models, the MSE prediction function (3.7) is not predictive consistent. This is not surprising because MSE analysis is often used simply as a criterion for evaluating the forecasting errors of point predictions without regard for functional form (e.g. Baillie (1981)). Indeed, abstracting from nonlinear functional forms is regarded as a virtue of the technique and MSE formula have been derived for quite general dynamic models (Baillie (1980)). Advocates of the MSE approach might respond to this failure to converge to zero information loss in large samples by substituting a quadratic loss function for $\bar{I}\left(f, f^{*}\right)$.

\footnotetext{
${ }^{4}$ The expected value of $I\left(f, f^{*}\right)$ over the distribution of $\hat{\theta}_{d}$ is typically about as difficult to derive as is the expected value of $\hat{\theta}_{d}$ itself. For example, $\bar{I}\left(f\right.$, plik $\left.^{a}\right)$ can be derived precisely for linear-normal models and models with nonlinearities in variables as in Cooley, Parke, and Chib (1989).

${ }^{5}$ The notion of $m$ going to infinity prior to the forecast period requires that we contemplate letting the sampling experiment run for longer periods before stopping data collection to make a prediction.
} 
Among predictive consistent functions, the first-order predictive efficiency is the scalar $\lambda_{1}\left(f, f^{*}\right)$ in the expansion

$$
\bar{I}\left(f, f^{*}\right)=m^{-1} \lambda_{1}\left(f, f^{*}\right)+o\left(m^{-1}\right) .
$$

We can derive the first-order predictive efficiency for the CEQ function under fairly general assumptions:

Proposition 1: We assume that: $(i)$ the derivatives to order three of $g\left(y_{f} ; \theta\right)$ with respect to $\theta$ exist, (ii) the derivatives to order two are bounded by integrable functions, and (iii) the third derivatives are uniformly bounded by a function with finite expectation. Then the CEQ first-order prediction efficiency is given by:

$$
\lambda_{1}(f, \hat{f})=-\frac{1}{2} \operatorname{tr}\left[V\left(\hat{\theta}_{d}\right) E_{Y}\left(H\left(y_{f} ; \theta\right)\right)\right],
$$

where the variance-covariance matrix of $m^{1 / 2}\left(\hat{\theta}_{d}-\theta\right)$ converges to $V\left(\hat{\theta}_{d}\right)$ and $E_{Y}\left(H\left(y_{f} ; \theta\right)\right)$ is the expectation over $Y$ of $H\left(y_{f} ; \theta\right)$.

Proof: Under these standard assumptions, we can expand

$$
\bar{I}(f, \hat{f})=\int_{\Theta} \int_{Y}\left(g\left(y_{f} \mid \theta\right)-g\left(y_{f} \mid \hat{\theta}_{d}\right)\right) f\left(y_{f} \mid \theta\right) f\left(\hat{\theta}_{d} \mid \theta\right) d y_{f} d \hat{\theta}_{d}
$$

as the sum of two expressions. The first,

$$
-\int_{\Theta} \int_{Y} \nabla\left(y_{f} ; \theta\right)\left(\hat{\theta}_{d}-\theta\right) f\left(y_{f} ; \theta\right) f\left(\hat{\theta}_{d} ; \theta\right) d y_{f} d \hat{\theta}_{d}
$$

equals zero because $\nabla\left(y_{f} ; \theta\right)$ is independent of $\hat{\theta}_{d}-\theta$ and $\int_{Y} \nabla\left(y_{f} ; \theta\right) f\left(y_{f} \mid \theta\right) d y_{f}$ $=0$. The second,

$$
-\frac{1}{2} \int_{\Theta} \int_{Y}\left(\hat{\theta}_{d}-\theta\right)^{\prime} H\left(y_{f} ; \theta^{*}\right)\left(\hat{\theta}_{d}-\theta\right) f\left(y_{f} \mid \theta\right) f\left(\hat{\theta}_{d} \mid \theta\right) d y_{f} d \hat{\theta}_{d},
$$

where $\theta^{*}$ is between $\theta$ and $\hat{\theta}_{d}$, converges (multiplied by $m$ ) to (4.5). Q.E.D.

We now turn our attention toward efficiency improvements that can be secured by accounting for parameter uncertainty. Proposition 1 states that ignoring parameter uncertainty leads to $\bar{I}(f, \hat{f})=O\left(\mathrm{~m}^{-1}\right)$, and it will turn out that the best improvement generally available is $O\left(\mathrm{~m}^{-2}\right)$. This bound on relative prediction efficiency is meaningful (as are the well known bounds on estimation efficiency) only if the class of alternatives is restricted by suitable regularity conditions. We gain some insight into establishing appropriate regularity conditions by considering a simple, but compelling example of superefficiency. The superefficiency example motivates us to require that any efficiency gain occur over a neighborhood $N$ in the parameter space rather than for just certain true parameters. Given this last assumption, we show in Proposition 2 that the largest possible efficiency improvement is $O\left(\mathrm{~m}^{-2}\right)$. We then contemplate the efficiency improvement achieved by the particular case $\operatorname{plik}^{a}\left(y_{f} \mid \hat{\theta}_{d}\right)$. 
We begin by formally defining the predictive efficiency of $f^{*}\left(y_{f} \mid \hat{\theta}_{d}\right)$ relative to $f\left(y_{f} \mid \hat{\theta}_{d}\right)$ as

$$
\bar{I}\left(\hat{f}, f^{*}\right)=-\int h\left(y_{f} ; \hat{\theta}_{d}\right) f\left(y_{f} \mid \theta\right) f\left(\hat{\theta}_{d} \mid \theta\right) d y_{f} d \hat{\theta}_{d},
$$

where $h\left(y_{f} ; \hat{\theta}_{d}\right)$ is defined as:

$$
h\left(y_{f} ; \hat{\theta}_{d}\right)=g^{*}\left(y_{f} ; \hat{\theta}_{d}\right)-g\left(y_{f} ; \hat{\theta}_{d}\right) .
$$

Note that the efficiency measures are additive in the sense that $\bar{I}\left(f, f^{*}\right)=$ $\bar{I}(f, \hat{f})+\bar{I}\left(\hat{f}, f^{*}\right)$. The second-order relative predictive efficiency is the scalar $\lambda_{2}\left(\hat{f}, f^{*}\right)$ in the expansion

$$
\bar{I}\left(\hat{f}, f^{*}\right)=m^{-2} \lambda_{2}\left(\hat{f}, f^{*}\right)+o\left(m^{-2}\right) .
$$

The regularity conditions we will introduce rule out certain instances of superefficiency. Consider the example of a prediction function $f^{*}\left(y_{f} \mid \hat{\theta}_{d}\right)=$ $f\left(y_{f} \mid \theta^{a}\right)$, where $\theta^{a}$ is a fixed element of $\Theta$. This choice entails zero information loss if $\theta$ happens to equal $\theta^{a}$, but not for any other true parameters. It fails to attain even predictive consistency for $\theta \neq \theta^{a}$ because $f^{*}\left(y_{f} \mid \hat{\theta}_{d}\right)=f\left(y_{f} \mid \alpha^{a}\right)$ for all $\hat{\theta}_{d}$ regardless of the actual true parameters. To rule out such cases, we require that the advantage of $f^{*}\left(y_{f} \mid \hat{\theta}_{d}\right)$ over $f\left(y_{f} \mid \hat{\theta}_{d}\right)$ be reasonably uniform for true parameters in a neighborhood $N$ (that does not shrink with increasing $m$ ). We incorporate this requirement via the average of $\bar{I}\left(\hat{f}, f^{*}\right)$ over all true parameters $\tilde{\theta}$ in $N$, which we write as

$$
\bar{I}_{N}=-\int_{N} \int_{\Theta}\left[\int_{Y} h\left(y_{f} ; \hat{\theta}_{d}\right) f\left(y_{f} \mid \tilde{\theta}\right) d y_{f}\right] f\left(\hat{\theta}_{d} \mid \tilde{\theta}\right) d \hat{\theta}_{d} d \tilde{\theta} .
$$

Our strategy will be to show that $\bar{I}_{N}$ can be negative, favoring $f^{*}$ over $\hat{f}$, only if $\bar{I}\left(\hat{f}, f^{*}\right)=O\left(m^{-2}\right)$ almost everywhere in $N$.

We begin by showing that insisting on an efficiency improvement $\bar{I}\left(\hat{f}, f^{*}\right)<0$ imposes restrictions on candidate functions $h\left(y_{f} ; \hat{\theta}_{d}\right)$. In particular there must exist an $\alpha$ such that

$$
\int\left[h\left(y_{f} ; \tilde{\theta}\right)\right]^{2} f\left(y_{f} \mid \tilde{\theta}\right) d y_{f}=O\left(m^{-\alpha}\right)
$$

and

$$
\int\left[h\left(y_{f} ; \tilde{\theta}\right)\right]^{k} f\left(y_{f} \mid \tilde{\theta}\right) d y_{f}=o\left(m^{-\alpha}\right), \quad k>2 .
$$

We can interpret these conditions in terms of the unit integral requirement $\int_{Y} \exp \left(h\left(y_{f} ; \hat{\theta}_{d}\right)\right) f\left(y_{f} \mid \hat{\theta}_{d}\right) d y_{f}=1$, which can be expanded as

$$
\int_{Y}\left[h\left(y_{f} ; \hat{\theta}_{d}\right)+\frac{1}{2}\left(h\left(y_{f} ; \hat{\theta}_{d}\right)\right)^{2}+\cdots\right] f\left(y_{f} \mid \hat{\theta}_{d}\right) d y_{f}=0 .
$$

Typically, $h\left(y_{f} ; \hat{\theta}_{d}\right)$ will be proportional to $V\left(\hat{\theta}_{d}\right)$ so that $m$ times $h\left(y_{f} ; \hat{\theta}_{d}\right)$ will converge to a nondegenerate limit. In that case, (4.10) and (4.11) will be 
satisfied for $\alpha=2$ and the first two terms of (4.12) will be of opposite signs and will dominate the remaining terms. To obtain this result formally, we consider an expansion

$$
\bar{I}_{N}=m^{-\delta} \lambda+o\left(m^{-\delta}\right) .
$$

Proposition 2 establishes that, for $\lambda$ to be negative, favoring $f^{*}$ over $\hat{f}, \alpha$ must satisfy $\alpha \geqslant 2$.

Two technical assumptions bound the moments of the gradient function. To the usual assumption

$$
\int_{Y} \nabla\left(y_{f} ; \theta\right)^{\prime} \nabla\left(y_{f} ; \theta\right) f\left(y_{f} \mid \theta\right) d y_{f}=O(1)
$$

that the variance exists, we add the requirement

$$
\int_{Y}\left(\nabla\left(y_{f} ; \theta\right)^{\prime} \nabla\left(y_{f} ; \theta\right)+H\left(y_{f} ; \theta\right)\right)^{2} f\left(y_{f} \mid \theta\right) d y_{f}=O(1)
$$

that the fourth moment exists as well. This is important because (4.12) shows that the square of $h\left(y_{f} ; \hat{\theta}_{d}\right)$ will be important and $h\left(y_{f} ; \hat{\theta}_{d}\right)$ involves the square of the gradient.

Proposition 2: The parameter $\lambda$ in (4.13) will be unambiguously positive unless (4.10) and (4.11) are satisfied for $\alpha \geqslant 2$ almost everywhere in $N$.

Proof: For notational simplicity we will use a scalar $\theta$. To evaluate the integral (4.9) over the Cartesian product $N \times \Theta$, we will integrate first over $N$ and then over $\Theta$. For a given $\hat{\theta}_{d} \in \Theta$, the integral over $\tilde{\theta} \in N$ can be written as the sum of two terms:

$$
\begin{aligned}
& -\int_{N}\left[\int_{Y} h\left(y_{f} ; \hat{\theta}_{d}\right) f\left(y_{f} \mid \hat{\theta}_{d}\right) d y_{f}\right] f\left(\hat{\theta}_{d} \mid \tilde{\theta}\right) d \tilde{\theta} \\
& -\quad \int_{N}\left\{\int_{Y} h\left(y_{f} ; \hat{\theta}_{d}\right)\left[e^{g\left(y_{f} ; \tilde{\theta}\right)-g\left(y_{f} ; \hat{\theta}_{d}\right)}-1\right] f\left(y_{f} \mid \hat{\theta}_{d}\right) d y_{f}\right\} f\left(\hat{\theta}_{d} \mid \tilde{\theta}\right) d \tilde{\theta}
\end{aligned}
$$

We can rewrite the first term in (4.16) as

$$
\int_{Y} h\left(y_{f} ; \hat{\theta}_{d}\right) f\left(y_{f} \mid \hat{\theta}_{d}\right) d y_{f} \int_{N} f\left(\hat{\theta}_{d} \mid \tilde{\theta}\right) d \tilde{\theta}
$$

where the second factor can be denoted $P\left(N \mid \hat{\theta}_{d}\right){ }^{6}$ The first factor in (4.17) is unambiguously nonnegative because $\int \log \left(\pi / \pi^{*}\right) \pi \geqslant 0$ for any densities $\pi$ and $\pi^{*}$ with equality only for $\pi^{*}=\pi$ almost everywhere. Expanding the exponential

\footnotetext{
${ }^{6}$ The notation $P\left(N \mid \hat{\theta}_{d}\right)$ conditioning on $\hat{\theta}_{d}$ is not completely well defined because $\tilde{\theta}$ is not a random variable. It does, however, furnish a convenient shorthand description of the process of integrating over $N$. The same consideration motivates our later use of the notation $E_{N}(\cdot)$.
} 
function in square brackets in the second term in (4.16) yields:

$$
\begin{aligned}
& -\int_{Y} h\left(y_{f} ; \hat{\theta}_{d}\right) \nabla\left(y_{f} ; \hat{\theta}_{d}\right) f\left(y_{f} \mid \hat{\theta}_{d}\right) d y_{f} \int_{N}\left(\hat{\theta}_{d}-\tilde{\theta}\right) f\left(\hat{\theta}_{d} ; \tilde{\theta}\right) d \tilde{\theta} \\
& -\frac{1}{2} \int_{Y} h\left(y_{f} ; \hat{\theta}_{d}\right)\left[\nabla\left(y_{f} ; \hat{\theta}_{d}\right)^{2}+H\left(y_{f} ; \hat{\theta}_{d}\right)\right] f\left(y_{f} \mid \hat{\theta}_{d}\right) d y_{f} \\
& \quad \times \int_{N}\left(\hat{\theta}_{d}-\tilde{\theta}\right)^{2} f\left(\hat{\theta}_{d} ; \tilde{\theta}\right) d \tilde{\theta}
\end{aligned}
$$

plus terms involving third and higher powers of $\hat{\theta}_{d}-\tilde{\theta}$.

We can now combine (4.12), (4.17), and (4.18) to write (4.16) as the product of $P\left(N \mid \hat{\theta}_{\dot{d}}\right)$ and

$$
\begin{aligned}
& V_{Y}(\hat{h})-\operatorname{Cov}_{Y}(\hat{h}, \hat{\nabla}) \cdot E_{N}\left(\hat{\theta}_{d}-\tilde{\theta} \mid \hat{\theta}_{d}\right) \\
& \quad-\operatorname{Cov}_{Y}\left(\hat{h}, \hat{\nabla}^{2}+\hat{H}\right) \cdot E_{N}\left(\left(\hat{\theta}_{d}-\tilde{\theta}\right)^{2} \mid \hat{\theta}_{d}\right),
\end{aligned}
$$

where $E_{N}\left(\hat{\theta}_{d}-\tilde{\theta} \mid \hat{\theta}_{d}\right)$ and $E_{N}\left(\left(\hat{\theta}_{d}-\tilde{\theta}\right)^{2} \mid \hat{\theta}_{d}\right)$ denote the order $m^{-1}$ terms in the asymptotic conditional mean and variance of $\hat{\theta}_{d}-\tilde{\theta}$ over $N$ and $\operatorname{Cov}_{Y}(\cdot)$ and $V_{Y}(\cdot)$ denote integrals over $y_{f}$. The covariance inequality together with (4.10), (4.14), and (4.15) implies that $\operatorname{Cov}_{Y}(\hat{h}, \hat{\nabla})=O\left(m^{-\alpha / 2}\right)$ and $\operatorname{Cov}_{Y}\left(\hat{h}, \hat{\nabla}^{2}+\hat{H}\right)=$ $O\left(m^{-\alpha / 2}\right)$. The two elements of (4.19) involving these factors are thus both $O\left(m^{-\alpha / 2-1}\right)$ while the unambiguously positive element $V_{Y}(\hat{h})$ is $O\left(m^{-\alpha}\right)$. From this, we deduce that the largest term in an expansion of (4.16) will be unambiguously positive unless $\alpha \geqslant 2$.

Integrating (4.16) over $\hat{\theta}_{d} \in \Theta$ will then produce (4.9). This last integral will be dominated by the unambiguously positive instances of $\alpha<2$ if these have measure greater than zero over $\hat{\theta}_{d} \in \Theta$.

Q.E.D.

The proof of Proposition 2 gives some guidance in constructing $h\left(y_{f} ; \hat{\theta}_{d}\right)$. The unambiguously nonnegative term $V_{Y}(\hat{h})$ should be as small as possible and the covariances $\operatorname{Cov}_{Y}(\hat{h}, \hat{\nabla})$ and $\operatorname{Cov}_{Y}\left(\hat{h}, \hat{\nabla}^{2}+\hat{H}\right)$ should be as large as possible. The functions $\nabla\left(y_{f} ; \hat{\theta}_{d}\right)$ and $\nabla\left(y_{f} ; \hat{\theta}_{d}\right)^{2}+H\left(y_{f} ; \hat{\theta}_{d}\right)$ are clear candidates to form $h\left(y_{f} ; \hat{\theta}_{d}\right)$ under these criteria. The asymptotic predictive likelihood function combines these two functions, weighting $\nabla\left(y_{f} ; \hat{\theta}_{d}\right)$ by the asymptotic bias and weighting $\nabla\left(y_{f} ; \hat{\theta}_{d}\right)^{2}+H\left(y_{f} ; \hat{\theta}_{d}\right)$ by the asymptotic variance.

A more direct approach to calculating the second-order relative efficiency of $\operatorname{plik}^{a}\left(y_{f} \mid \hat{\theta}_{d}\right)$ and other prediction functions is possible if the conditions in Proposition 2 are strengthened slightly. The main conclusion of Proposition 2 is that reasonable candidate functions $h\left(y_{f} ; \hat{\theta}_{d}\right)$ will be well behaved after multiplication by $m$. In practice, $h\left(y_{f} ; \hat{\theta}_{d}\right)$ will generally be constructed by weighting a function of $y_{f}$ by either the variance or bias of $\hat{\theta}_{d}$, both of which are proportional to $m^{-1}$.

Proposition 3: Assume that ( $i)$ the derivatives to order three of $m h\left(y_{f} ; \theta\right)$ with respect to $\theta$ exist (we will denote derivatives by subscripts, e.g. $h_{\theta}\left(y_{f} ; \theta\right)$ ), (ii) the 
derivatives to order two of $m h\left(y_{f} ; \theta\right)$ are bounded by integrable functions, (iii) the third derivatives of $\operatorname{mh}\left(y_{f} ; \theta\right)$ are uniformly bounded by a function with finite expectation, and (iv) (4.10) and (4.11) are satisfied for $\alpha=2$. Then

$$
\lambda_{2}\left(\hat{f}, f^{*}\right)=\frac{1}{2} \underline{h}^{2}-\psi \underline{h}_{\theta}-\frac{1}{2} \operatorname{tr}\left[V\left(\hat{\theta}_{d}\right) \underline{h}_{\theta \theta}\right]
$$

where

$$
\begin{aligned}
& \underline{h}^{2}=\lim _{m \rightarrow \infty} \int_{Y} m^{2} h\left(y_{f} ; \theta\right)^{2} f\left(y_{f} \mid \theta\right) d y_{f}, \\
& \underline{h}_{\theta}=\lim _{m \rightarrow \infty} \int_{Y} m h_{\theta}\left(y_{f} ; \theta\right) f\left(y_{f} \mid \theta\right) d y_{f}, \\
& \underline{h}_{\theta \theta}=\lim _{m \rightarrow \infty} \int_{Y} m h_{\theta \theta}\left(y_{f} ; \theta\right) f\left(y_{f} \mid \theta\right) d y_{f}, \quad \text { and } \\
& m^{-1} \psi \text { is the } O\left(m^{-1}\right) \text { bias in } \hat{\theta}_{d} .
\end{aligned}
$$

Proof: By expanding $h\left(y_{f} ; \theta\right)$, we can write

$$
-m^{2} \int_{\Theta} \int_{Y} h\left(y_{f} ; \hat{\theta}_{d}\right) f\left(y_{f} \mid \theta\right) f\left(\hat{\theta}_{d} \mid \theta\right) d y_{f} d \hat{\theta}_{d}
$$

as the sum of three terms

$$
\begin{aligned}
& -m^{2} \int_{Y} h\left(y_{f} ; \theta\right) f\left(y_{f} \mid \theta\right) d y_{f}, \\
& -m^{2} \int_{\Theta} \int_{Y} h_{\theta}\left(y_{f} ; \theta\right)\left(\hat{\theta}_{d}-\theta\right) f\left(y_{f} \mid \theta\right) f\left(\hat{\theta}_{d} \mid \theta\right) d y_{f} d \hat{\theta}_{d}, \\
& -\frac{1}{2} m^{2} \int_{\Theta} \int_{Y}\left(\hat{\theta}_{d}-\theta\right)^{\prime} h_{\theta \theta}\left(y_{f} ; \theta^{*}\right)\left(\hat{\theta}_{d}-\theta\right) f\left(y_{f} \mid \theta\right) f\left(\hat{\theta}_{d} \mid \theta\right) d y_{f} d \hat{\theta}_{d},
\end{aligned}
$$

where $\theta^{*}$ is between $\theta$ and $\hat{\theta}_{d}$. The first term can be approximated by $\frac{1}{2} \underline{h}^{2}$ via the unit integral requirement (4.12) and assumption (iv). The other two terms converge in probability to the terms in (4.20).

Q.E.D.

For the particular prediction function $\operatorname{plik}^{a}\left(y_{f} \mid \hat{\theta}_{d}\right)$, the conclusion of Proposition 3 can be rewritten to avoid integrating the square of $h$. We record these results as:

Proposition 4: (a) If the bias in $\hat{\theta}_{d}$ is $o\left(m^{-1}\right)$ ( perhaps, because $\hat{\theta}_{d}$ is corrected for bias $)$, then $\lambda_{2}\left(\hat{f}, \mathrm{plik}^{a}\right)$ can be written as:

$$
\begin{aligned}
-\frac{1}{8} \operatorname{vec}\left(H_{d}^{-1}\right)^{\prime}\left\{E _ { Y } \left[\left(\operatorname{vec} H_{f}\right)\right.\right. & \left.\left(\operatorname{vec} H_{f}\right)^{\prime}\right]-E_{Y}\left[\nabla_{f}^{\prime} \otimes H_{f} \otimes \nabla_{f}\right] \\
+ & \left.2 E_{Y}\left[H_{f}\right]_{\theta \theta}-E_{Y}\left[\left(\nabla_{f}^{\prime} \otimes \nabla_{f}^{\prime}\right) \nabla_{f}\right]_{\theta}\right\} \operatorname{vec}\left(H_{d}^{-1}\right),
\end{aligned}
$$

where we are letting the arguments of $H_{d}, H_{f}$, and $\nabla_{f}$ be implicit for notational simplicity. 
(b) If, in addition, $E_{Y}\left[H_{f}\right]$ and $E_{Y}\left[\left(\nabla_{f}^{\prime} \otimes \nabla_{f}^{\prime}\right) \otimes \nabla_{f}\right]$ are constant over $\theta$, then

$$
\lambda_{2}\left(\hat{f}, \operatorname{plik}^{a}\right)=-\frac{1}{8} E_{Y}\left[\left(\operatorname{tr}\left(H_{d}^{-1} H_{f}\right)\right)^{2}\right]+\frac{1}{8} E_{Y}\left[\nabla_{f} H_{d}^{-1} H_{f} H_{d}^{-1} \nabla_{f}^{\prime}\right]
$$

(c) If $H\left(y_{f} ; \theta\right)$ is globally negative semi-definite, then $\lambda_{2}\left(\hat{f}, \mathrm{plik}^{a}\right)$ is unambiguously negative.

\section{Proof: Appendix B.}

Proposition 4 applies to the classic location parameter prediction problem where $f\left(y_{f} \mid \theta\right)$ is of the form $q\left(y_{f}-\theta\right)$ so that we know the form of the density for $y_{f}$, but not its location. An example, considered in Section 6 of this paper, is a regression model with fat-tailed error terms. Part (c) shows an unambiguous efficiency gain if $\log \left(q\left(y_{f}-\theta\right)\right)$ is any symmetric convex function (e.g. $y_{f}$ follows a $t$ distribution). We consider this case and other examples applying Propositions 3 and 4 in Section 6.

\section{INEFFICIENT STATISTICS}

In practical problems, interest will often center on data period parameter estimates that are consistent and asymptotically normal, but not asymptotically efficient. (Consider, for example, two stage least-squares.) Let $\tilde{\theta}_{d}$ be such an estimate with

$$
m^{1 / 2}\left(\tilde{\theta}_{d}-\theta\right) \stackrel{d}{\rightarrow} N\left(O, V\left(\tilde{\theta}_{d}\right)\right),
$$

where $V\left(\tilde{\theta}_{d}\right)-V\left(\hat{\theta}_{d}\right)$ is a positive semi-definite matrix. Let $H_{d+f}$ denote $V_{m}^{-1}+$ $H_{f}$, where $m V_{m}$ is a consistent estimate of $V\left(\tilde{\theta}_{d}\right)$. We can then extend our asymptotic predictive likelihood definition to cover prediction functions based on $\tilde{\theta}_{d}$ :

$$
\operatorname{plik}^{c}\left(y_{f} \mid y_{d}\right)=f\left(y_{f} \mid \tilde{\theta}_{d}\right) \cdot \exp \left\{w_{1}\left(y_{f} ; \tilde{\theta}_{d}\right)+w_{2}\left(y_{f} ; \tilde{\theta}_{d}\right)\right\}
$$

where

$$
\begin{aligned}
& w_{1}\left(y_{f} ; \tilde{\theta}_{d}\right)=-\frac{1}{2} \nabla\left(y_{f} ; \tilde{\theta}_{d}\right) H\left(y_{d+f} ; \tilde{\theta}_{d}\right)^{-1} \nabla\left(y_{f} ; \tilde{\theta}_{d}\right)^{\prime}, \\
& w_{2}\left(y_{f} ; \tilde{\theta}_{d}\right)=\nabla\left(y_{f} ; \tilde{\theta}_{d}\right) \psi\left(\tilde{\theta}_{d}\right)-\frac{1}{2} \operatorname{tr}\left[H\left(y_{f} ; \tilde{\theta}_{d}\right) H\left(y_{d} ; \tilde{\theta}_{d}\right)^{-1}\right] .
\end{aligned}
$$

This definition is motivated further in Appendix A. This prediction function possesses many of the important features of (3.1), taking due account of the fact that the estimate $\tilde{\theta}_{d}$ is not asymptotically efficient.

In particular, plik ${ }^{c}\left(y_{f} \mid \tilde{\theta}_{d}\right)$ may well secure an efficiency gain over the corresponding plug-in function $\tilde{f}=f\left(y_{f} \mid \tilde{\theta}_{d}\right)$ also based on the inefficient estimates $\tilde{\theta}_{d}$. In terms of first-order efficiency, direct extensions of Propositions 1 and 2 show that $\lambda_{1}\left(f, \operatorname{plik}^{c}\right)=\lambda_{1}(f, \tilde{f})$ and that $\operatorname{plik}^{c}\left(y_{f} \mid y_{d}\right)$ is first-order inefficient relative 
to $\operatorname{plik}^{a}\left(y_{f} \mid y_{d}\right)$ to the extent that

$$
\lambda_{1}(\hat{f}, \tilde{f})=-\frac{1}{2} \operatorname{tr}\left[E_{Y}\left(H\left(y_{f} ; \theta\right)\right)\left(V\left(\tilde{\theta}_{d}\right)-V\left(\hat{\theta}_{d}\right)\right)\right]
$$

is positive. Extensions of Propositions 3 and 4, on the other hand, would suggest that the CEQ $f\left(y_{f} \mid \tilde{\theta}_{d}\right)$ may be second-order inefficient relative to $\operatorname{plik}^{c}\left(y_{f} \mid \hat{\theta}_{d}\right)$. Thus, from a practical standpoint, if sufficient motivation exists to favor calculation of only inefficient parameter estimates, $\operatorname{plik}^{c}\left(y_{f} \mid \tilde{\theta}_{d}\right)$ still incorporates a useful adjustment for parameter uncertainty.

\section{EXAMPLES}

The definition of asymptotic predictive likelihood and the concept of predictive efficiency developed above are useful only to the extent that they sharpen our understanding of practical prediction problems. In this section we consider examples that extend well known results for linear-normal models to models with nonnormal disturbances and to ARCH models.

EXAMPLE 1 (Linear-Normal Model): Before considering more complex models, it is helpful to examine the asymptotic efficiency concepts for a linear regression model because Definitions 1 and 2 coincide and exact information losses can be computed. We write that model as

$$
y_{i}=x_{i} \beta+\varepsilon_{i}, \quad \varepsilon_{i} \sim N\left(0, \sigma^{2}\right),
$$

for regressors $x_{i}$, parameters $\beta$, and known $\sigma^{2}$. Cooley, Parke, and Chib (1989) show that, for a single future observation, $\operatorname{plik}^{a}\left(y_{f} \mid \hat{\theta}_{d}\right) \propto \exp \left(-\frac{1}{2} \varepsilon_{f}^{2} /\left(\sigma^{2}+\tau^{2}\right)\right)$, where the variance component $\tau^{2}=\sigma^{2} x_{f}\left(x_{d}^{\prime} x_{d}\right)^{-1} x_{f}^{\prime}$ corrects for parameter uncertainty. The comparison between the two efficiencies $\bar{I}(f, \hat{f})=\frac{1}{2} \tau^{2} / \sigma^{2}$ and $\bar{I}\left(f\right.$, plik $\left.^{a}\right)=\frac{1}{2} \log \left(1+\tau^{2} / \sigma^{2}\right)$ can be put into the present framework by expanding the second of these as $\frac{1}{2} \tau^{2} / \sigma^{2}-\frac{1}{4}\left(\tau^{2} / \sigma^{2}\right)^{2}+\cdots$. The firstorder asymptotic efficiencies equal $\frac{1}{2} \lim _{m \rightarrow \infty} m \tau^{2} / \sigma^{2}$ in both cases. Correcting for parameter uncertainty secures the second-order efficiency gain $\frac{1}{4} \lim _{m \rightarrow \infty} m^{2}\left(\tau^{2} / \sigma^{2}\right)^{2}$, which is reflected in the curvature of the log function. That efficiency gain is likely to be most important for difficult forecasting problems where the first-order efficiency loss is also important.

EXAmple 2 (Nonnormal Model): Suppose that the errors are drawn from a $t$ distribution with $\nu$ degrees of freedom, where $\sigma$ and $\nu$ are known. (For the variance to exist we require that $\nu>2$.) The predictive likelihood function takes account of the relatively fat tails in the $t$ distribution. If we let $\zeta$ denote $\left(y_{m+1}-x_{m+1} \hat{\beta}_{d}\right) / \sigma$, then for a single future observation

$$
\operatorname{plik}^{a}\left(y_{f} \mid \hat{\beta}_{d}\right) \propto \frac{1}{\sigma}\left(1+\zeta^{2} / \nu\right)^{-(\nu+1) / 2} \cdot \exp \left\{w_{1}\left(y_{f} ; \hat{\theta}_{d}\right)+w_{2}\left(y_{f} ; \hat{\theta}_{d}\right)\right\}
$$


where

$$
w_{1}\left(y_{f} ; \hat{\theta}_{d}\right)=-\frac{1}{2} \frac{(\nu+1)^{2}}{\nu^{2}} \frac{x_{f} H_{d+f}^{-1} x_{f}^{\prime}}{\left(1+\zeta^{2} / \nu\right)^{2}} \zeta^{2}
$$

and

$$
w_{2}\left(y_{f} ; \hat{\theta}_{d}\right)=\frac{(\nu+1)}{\nu} \frac{x_{f} H_{d}^{-1} x_{f}^{\prime}}{\left(1+\zeta^{2} / \nu\right)^{2}}\left(1-\zeta^{2} / \nu\right) .
$$

The correction for parameter uncertainty $w_{1}\left(y_{f} ; \hat{\theta}_{d}\right)$ increases the dispersion of the plik by adding to the density in the tails, where $\zeta^{2}$ is greatest. Relative to the linear-normal model in Example 1, however, the true density already has fat tails, and the denominator $\left(1+\zeta^{2} / \nu\right)^{2}$ in $w_{1}\left(y_{f} ; \hat{\beta}_{d}\right)$ moderates the extent of the correction in the extreme tails. The term $w_{2}\left(y_{f} ; \hat{\beta}_{d}\right)$ adds a lesser correction for a nonconstant second derivative matrix.

Proposition 4 provides the information efficiency calculations for this model. If we let $\Gamma$ denote $\lim _{m \rightarrow \infty} m x_{f}\left(x_{d}^{\prime} x_{d}\right)^{-1} x_{f}^{\prime}$, then

$$
\lambda_{1}(f, \hat{f})=\frac{1}{2} \frac{\nu(\nu+1)}{(\nu-2)(\nu+3)} \Gamma .
$$

This figure ranges from the value of $\frac{1}{2} \Gamma$ for Example $1(\nu=\infty)$ to $\infty$ for $\nu=2$, revealing the extent to which fatter tails (smaller $\nu$ ) lead to a more difficult forecasting problem. The second-order relative efficiency

$$
\begin{aligned}
\lambda_{2}\left(\hat{f}, \operatorname{plik}^{a}\right)= & -\frac{1}{8} \frac{(\nu+1)^{2}}{(\nu-2)^{2}} \frac{(\nu+6)(\nu+4)(\nu+2) \nu}{(\nu+7)(\nu+5)(\nu+3)(\nu+1)} \\
& \times\left[1+\frac{(\nu-1)}{(\nu+6)}-\nu \frac{3+6 /(\nu+4)}{(\nu+6)^{2}}\right] \Gamma^{2}
\end{aligned}
$$

is clearly negative so that the predictive likelihood correction for parameter uncertainty lowers the information loss.

Unknown error distribution parameters such as $\sigma^{2}$ and $\nu$ also present interesting forecasting problems. If $\sigma^{2}$ in Example 1 is unknown, a direct analysis of the sampling distribution of $\zeta=\left(y_{f}-x_{f} \hat{\beta}_{d}\right) / s$, where $s$ is the sample period estimate of $\sigma$, shows the appropriate prediction function to be of the functional form of a $t$ distribution. Definition 1 yields precisely this result using a $\chi^{2}$ distribution for the sample variance $s^{2}$ (Cooley, Parke, and Chib (1989)). The advantage of $\operatorname{plik}^{a}\left(y_{f} \mid s^{2}\right)$ is that only $g\left(y_{f} ; \theta\right)$ and its derivatives are needed because Definition 2 is based (see Appendix A) on the asymptotically valid normal approximation $m^{1 / 2}\left(s^{2}-\sigma^{2}\right) \stackrel{d}{\rightarrow} N(0,2)$. For the simple model $y_{i} \sim$ $N\left(0, \sigma^{2}\right)$ that abstracts from uncertainty about $\beta$,

$$
\operatorname{plik}^{a}\left(y_{f} \mid s^{2}\right) \propto-\frac{1}{2} y_{f}^{2} / s^{2}-\frac{1}{4}(m+1)^{-1}\left(y_{f}^{2} / s^{2}-1\right)^{2} .
$$


This function is identical to the first two terms in a series expansion of the logarithm of a $t$ density for $y_{f}$, expanding $-\frac{1}{2}(\nu+1) \log \left(1+\left(y_{f}^{2} / s^{2}\right) / \nu\right)$ about $-\frac{1}{2}(\nu+1) \log (1+1 / \nu)$. The correction for parameter uncertainty in plik $^{a}\left(y_{f} \mid s^{2}\right)$ thus captures the essential features of a $t$ distribution.

The information efficiency calculations for this model are a special case of those for Example 3 below. We simply note here that $\operatorname{plik}^{a}\left(y_{f} \mid s^{2}\right)$ will be second-order efficient relative to the CEQ $f\left(y_{f} \mid s^{2}\right)$ to the extent that a $t$ distribution is more appropriate for $\left(y_{f}-x_{f} \hat{\beta}_{d}\right) / s$ than is a normal distribution.

ExAmple 3: The most interesting features of models with unknown error distribution parameters can be demonstrated in the context of the autoregressive conditional heteroskedasticity (ARCH) model. Following Engle (1982), we emphasize the essential aspects of this model using a simple ARCH model without regressors,

$$
y_{t} \sim N\left(0, \eta_{t}\right),
$$

where $\eta_{t}=z_{t} \alpha$ for $z_{t}=\left(1, y_{t-1}^{2}, \ldots, y_{t-p}^{2}\right)$ and $\alpha=\left(\alpha_{0}, \alpha_{1}, \ldots, \alpha_{p}\right)$. This model emphasizes the dispersion of the future density rather than its mean.

This model also illustrates the complications introduced by dependent observations. ${ }^{7}$ An extension of Definition 1 is not possible because, while one might consider enlarging the notation to condition on both a sufficient statistic $S_{d}$ and the last few values in $y_{d}$, this model does not admit sufficient statistics. Definition 2, on the other hand, presents no difficulties of this sort because the asymptotically sufficient maximum likelihood estimates are readily available and the corrections for parameter uncertainty $w_{1}\left(y_{f} ; \hat{\theta}_{d}\right)$ and $w_{2}\left(y_{f} ; \hat{\theta}_{d}\right)$ are constructed from density function derivatives that readily admit a dependence on the terminal values of $y_{d}$. The justification for such an extension rests largely on the efficiency results in Propositions 1, 2, 3, and 4, which are then conditional on the last few values in $y_{d}$. We can, for example, derive efficiency results for ARCH model predictions conditional on the realized value of $z_{m}$.

The predictive likelihood function again approximates the functional form of a $t$ distribution. ${ }^{8}$ For one period ahead (so that $f$ denotes $m+1$ ),

$$
\log \left(\operatorname{plik}^{a}\left(y_{f} \mid y_{d}, \hat{\alpha}_{d}\right)\right) \propto-\frac{1}{2} \frac{y_{f}^{2}}{\eta_{f}}-\frac{1}{8} \frac{z_{f} H_{d+f}^{-1} z_{f}^{\prime}}{\eta_{f}^{2}}\left\{\frac{y_{f}^{2}}{\eta_{f}}-1\right\}^{2} .
$$

In this approximation to a $t$ distribution, the "degrees of freedom"

$$
\nu_{m}=\frac{1}{2} \frac{\eta_{f}^{2}}{z_{f} H_{d+f}^{-1} z_{f}^{\prime}}
$$

\footnotetext{
${ }^{7}$ Another simple example is the AR(1) model (Cooley and Parke (1987)).

${ }^{8} \mathrm{We}$ omit the term $w_{2}\left(y_{f} ; \hat{\theta}_{d}\right)$ on two grounds. First, the asymptotic bias is not known for the ARCH model, making an analytic implementation impossible. Second, the two terms in $w_{2}\left(y_{f} \hat{\theta}_{d}\right)$ cancel for Example 2 and will largely offset in this case as well.

Our calculations are all conditional on the last few values of $y_{t}$. As Phillips (1979) notes, this introduces a minor dependency between the distribution of $\hat{\theta}_{d}$ and the last few values of $y_{t}$.
} 
will be proportional to the data period sample size because $H_{d+f}$ grows at rate $m$, but will also depend on the particular $z_{f}$ vector. That vector appears in both the numerator $\eta_{f}^{2}=\left(z_{f} \hat{\alpha}_{d}\right)^{2}$ and in the denominator, which essentially equals $V\left(z_{f} \hat{\alpha}_{d}\right)$. If, for example, the elements of $\hat{\alpha}_{d}$ are negatively correlated so that $H_{d+f}^{-1}$ has negative off-diagonal elements, $\nu_{m}$ will be smaller (and the correction for parameter uncertainty will be greater) for a vector $z_{f}$ with a single large element than for $z_{f}$ with more equally sized elements.

The formal information efficiency calculations also reflect the dependence of $\nu_{m}$ on $z_{f}$. The first-order information efficiency is

$$
\lambda_{1}(f, \hat{f})=\frac{1}{2} \nu_{\infty}^{-1}
$$

where $\nu_{\infty}=\lim _{m \rightarrow \infty} m^{-1} \nu_{m}$ depends on $z_{f}$. Proposition 3 shows that

$$
\lambda_{2}\left(\hat{f}, \operatorname{plik}^{a}\right)=-23 / 64 \nu_{\infty}^{-2} .
$$

The efficiency gain from correcting for parameter uncertainty thus depends on both the data period sample size and the particular vector $z_{f}$.

Predictive likelihood forecasts two or more periods ahead for an ARCH model recognize that the variance of $y_{m+2}$ depends on the realization of $y_{m+1}$. This dynamic aspect of the problem is incorporated into

$$
\log \left(\operatorname{plik}^{a}\left(y_{f} \mid y_{d}, \hat{\alpha}_{d}\right)\right) \propto-\frac{1}{2} \sum_{i=m+1}^{m+n} \frac{y_{m+i}^{2}}{\eta_{m+i}^{2}}-\frac{1}{8} \zeta^{\prime} z_{f} H_{d+f}^{-1} z_{f}^{\prime} \zeta,
$$

where $\zeta$ is the $n \times 1$ vector with elements $\zeta_{i}=\left(y_{m+i}^{2} / \eta_{m+i}-1\right)^{2} / \eta_{m+i}, i=$ $1, \ldots, n$. If $n=2$, then $y_{m+1}$ appears (via $z_{f}$ ) in both the $2 \times 2$ matrix $z_{f} H_{d+f}^{-1} z_{f}^{\prime}$ and in $\eta_{m+2}$. This joint predictive density for $y_{m+1}$ and $y_{m+2}$ thus makes the dispersion for $y_{m+2}$ a function of the entire range of values for $y_{m+1}$ weighted by their predictive likelihoods.

\section{CONCLUSIONS}

The asymptotic predictive likelihood approach analyzed in this paper is closely related to Monte Carlo forecasting approaches discussed in Section 2. Monte Carlo procedures account for parameter uncertainty by drawing coefficients from an asymptotic distribution. The predictive likelihood approach, on the other hand, suggests a correction to the forecasting density. The correction can be implemented easily using stochastic simulation with a weighting determined from the correction terms in Definition 2. Consequently, although the calculations in the examples seem cumbersome, implementing these prediction functions via simulation is quite feasible.

The information measure of predictive efficiency derived in Section 4 helps to identify the effects of specification and estimation on predictive accuracy. Predictive consistency requires the correct functional form for the model. First-order efficiency rests on the efficiency of the estimated parameters. Asymptotic estimation bias and corrections for parameter uncertainty affect 
second-order efficiency. This is one explanation of why parameter uncertainty appears not to matter much in many applications and is usually neglected.

Dept. of Economics and Simon School of Business, University of Rochester, Rochester, NY 14627, U.S.A.

\section{and}

Department of Economics, University of North Carolina, Chapel Hill, NC 27599, U.S.A.

Manuscript received September, 1986; final revision received March, 1990.

\section{APPENDIX A}

\section{Motivation for Predictive Likelihood Definitions}

In situations where sufficient reductions of the data do not exist, we can exploit the fact that well-behaved maximum likelihood estimates are asymptotically sufficient (Cox and Hinkley (1974, p. 307)). Replacing the sufficient statistics $S_{d}$ and $S_{d+f}$ in Definition 1 by the MLE's $\hat{\theta}_{d}$ and $\hat{\theta}_{d+f}$ leads to the alternative definition:

$$
\operatorname{plik}^{1}\left(y_{f} \mid \hat{\theta}_{d}\right)=f\left(y_{f}, \hat{\theta}_{d} \mid \hat{\theta}_{d+f}\right)=\frac{f\left(y_{f} \mid \theta\right) f\left(\hat{\theta}_{d} \mid \theta\right)}{f\left(\hat{\theta}_{d+f} \mid \theta\right)}
$$

where $f\left(\hat{\theta}_{d} \mid \theta\right)$ and $f\left(\hat{\theta}_{d+f} \mid \theta\right)$ are exact finite sample distributions of the MLE's. For econometric problems of any complexity these exact finite sample distributions are intractable. This consideration leads us to:

$$
\operatorname{plik}^{2}\left(y_{f} \mid \hat{\theta}_{d}\right)=\frac{f\left(y_{f} \mid \theta\right) f^{a}\left(\hat{\theta}_{d} \mid \theta\right)}{f^{a}\left(\hat{\theta}_{d+f} \mid \theta\right)}
$$

where $f^{a}(\cdot \mid \cdot)$ denotes an asymptotic density. $\hat{\theta}_{d+f}$ in the denominator of (A.2) is determined jointly by $\hat{\theta}_{d}$ and $y_{f}$ just as $S_{d+f}$ in Definition 1 is a function of $S_{d}$ and $y_{f}$. The predictive likelihood value measures the joint compatibility of $y_{f}$ and $\hat{\theta}_{d}$ with a common $\hat{\theta}_{d+f}$.

A further simplification eliminates the need to compute $\hat{\theta}_{d+f}$ for each possible $y_{f}$. We can relate $\hat{\theta}_{d}$ and $\hat{\theta}_{d+f}$ via

$$
\nabla\left(y_{d+f} ; \hat{\theta}_{d+f}\right)^{\prime}-\nabla\left(y_{d+f} ; \hat{\theta}_{d}\right)^{\prime}=H\left(y_{d+f} ; \theta\right)\left(\hat{\theta}_{d+f}-\hat{\theta}_{d}\right)+O_{p}\left(m^{-1 / 2}\right) .
$$

Using the fact that $\nabla\left(y_{d+f} ; \hat{\theta}_{d+f}\right)=0$ and $\nabla\left(y_{d} ; \hat{\theta}_{d}\right)=0$ (by the definitions of $\hat{\theta}_{d+f}$ and $\left.\hat{\theta}_{d}\right)$ and the independence of $y_{d}$ and $y_{f}$,

$$
\hat{\theta}_{d+f}=\hat{\theta}_{d}-\left[H\left(y_{d+f} ; \theta\right)\right]^{-1} \nabla\left(y_{f} ; \hat{\theta}_{d}\right)^{\prime}+O_{p}\left(m^{-3 / 2}\right) .
$$

We use the asymptotic distributions

$$
\begin{aligned}
& g^{a}\left(\hat{\theta}_{d} \mid \theta\right)=-\frac{1}{2}\left(\hat{\theta}_{d}-\psi_{d}-\theta\right)^{\prime} H\left(y_{d} ; \theta\right)\left(\hat{\theta}_{d}-\psi_{d}-\theta\right), \\
& g^{a}\left(\hat{\theta}_{d+f} \mid \theta\right)=-\frac{1}{2}\left(\hat{\theta}_{d+f}-\psi_{d+f}-\theta\right)^{\prime} H\left(y_{d+f} ; \theta\right)\left(\hat{\theta}_{d+f}-\psi_{d+f}-\theta\right),
\end{aligned}
$$

where $\psi$ is the $O\left(m^{-1}\right)$ bias and $\psi_{d+f}=\psi_{d}+o\left(m^{-1}\right)$. We match these quadratic forms with a Taylor series approximation to $g\left(y_{f} \mid \theta\right)$ :

$$
\begin{aligned}
g\left(y_{f} \mid \theta\right)= & g\left(y_{f} \mid \hat{\theta}_{d}\right)-\nabla\left(y_{f} ; \hat{\theta}_{d}\right)\left(\hat{\theta}_{d}-\theta\right) \\
& +\frac{1}{2}\left(\hat{\theta}_{d}-\theta\right)^{\prime} H\left(y_{f} ; \hat{\theta}_{d}\right)\left(\hat{\theta}_{d}-\theta\right)+O_{p}\left(m^{-3 / 2}\right) .
\end{aligned}
$$


Finally, substituting (A.4) into (A.5a), adding (A.6), and subtracting (A.5b) leaves three terms that are not constant with respect to $y_{f}$ :

$$
\frac{1}{2} \nabla\left(y_{f} ; \hat{\theta}_{d}\right) H^{-1}\left(y_{d+f} ; \theta\right) \nabla\left(y_{f} ; \hat{\theta}_{d}\right)^{\prime}+\nabla\left(y_{f} ; \hat{\theta}_{d}\right) \psi_{d}+\frac{1}{2}\left(\hat{\theta}_{d}-\theta\right)^{\prime} H\left(y_{f} ; \hat{\theta}_{d}\right)\left(\hat{\theta}_{d}-\theta\right) .
$$

The first is the basis for $w_{1}\left(y_{f} ; \hat{\theta}_{d}\right)$ using the estimate $H\left(y_{d+f} ; \hat{\theta}_{d}\right)$ of $H\left(y_{d+f} ; \theta\right)$. The second and third terms yield $w_{2}\left(y_{f} ; \hat{\theta}_{d}\right)$ via the estimate $-H\left(y_{d} ; \hat{\theta}_{d}\right)_{\tilde{\theta^{\prime}}}^{-1}$ of $E\left[\left(\hat{\theta}_{d}-\theta\right)\left(\hat{\theta}_{d}-\theta\right)^{\prime}\right]$.

Equation (5.1) requires a suitable joint estimate $\tilde{\theta}_{d+f}$ based on $\tilde{\theta}_{d}$ and $y_{f}$. The analytic tractability of the approximate density

$$
f^{a}\left(\tilde{\theta}_{d} \mid \theta\right) \propto \exp \left\{-\frac{1}{2}\left(\tilde{\theta}_{d}-\theta\right)^{\prime} V_{m}^{-1}\left(\tilde{\theta}_{d}-\theta\right)\right\}
$$

suggests letting the joint estimate $\tilde{\theta}_{d+f}$ be computed by maximizing

$$
g\left(\tilde{\theta}_{d}, y_{f} \mid \theta\right)=g\left(y_{f} \mid \theta\right)-\frac{1}{2}\left(\tilde{\theta}_{d}-\theta\right)^{\prime} V_{m}^{-1}\left(\tilde{\theta}_{d}-\theta\right)
$$

over $\theta$ for fixed $y_{f}$ and $\tilde{\theta}_{d}$. An asymptotic expansion similar to (A.3) shows that

$$
\tilde{\theta}_{d+f}=\tilde{\theta}_{d}+\left[V_{m}^{-1}-H\left(y_{f} ; \tilde{\theta}_{d}\right)\right]^{-1} \nabla\left(y_{f} ; \tilde{\theta}_{d}\right)^{\prime}+O_{p}\left(m^{-3 / 2}\right) .
$$

(5.1) follows from the previous analysis with (A.4) replaced by (A.7).

\section{APPENDIX B}

\section{Proof of Proposition 4}

We would like to apply Proposition 3 for

$$
h=\frac{1}{2} \nabla_{f} H_{d+f}^{-1} \nabla_{f}+\frac{1}{2} \operatorname{tr} H_{f} H_{d}^{-1} .
$$

For simplicity, we are letting the function arguments be implicit. Let $Z=\operatorname{vec} H_{d}^{-1}$, and note that $H_{d+f}^{-1}=H_{d}^{-1}+o_{p}\left(m^{-1}\right)$.

The term $\frac{1}{2} \underline{h}^{2}$ equals $\frac{1}{8} Z^{\prime} E[A] Z$, where

$$
A=\left(\nabla^{\prime} \otimes \nabla^{\prime}\right)(\nabla \otimes \nabla)+\nabla^{\prime} \otimes \nabla^{\prime} \otimes(\operatorname{vec} H)^{\prime}+(\operatorname{vec} H) \otimes \nabla \otimes \nabla+(\operatorname{vec} H)(\operatorname{vec} H)^{\prime} .
$$

Note that $\nabla H \nabla^{\prime} \nabla H \nabla=Z^{\prime}\left(\nabla^{\prime} \otimes \nabla^{\prime}\right)(\nabla \otimes \nabla) Z$ (Neudecker (1969))..$^{9}$ Generalizing Pfanzagl (1973, p. 997), $E\left[\left(\nabla^{\prime} \otimes \nabla^{\prime}\right) \nabla\right]_{\theta}$ can be written as

$$
\begin{array}{r}
E\left[\left(\nabla^{\prime} \otimes \nabla^{\prime}\right)(\nabla \otimes \nabla)\right]+E\left[\left(\nabla^{\prime} \otimes \nabla^{\prime}\right)(\operatorname{vec} H)^{\prime}\right] \\
+E[(\operatorname{vec} H)(\nabla \otimes \nabla)]+E\left[\nabla^{\prime} \otimes H \otimes \nabla\right]
\end{array}
$$

so that

$$
E[A]=E\left[(\operatorname{vec} H)(\operatorname{vec} H)^{\prime}\right]-E\left[\nabla^{\prime} \otimes H \otimes \nabla\right]+E\left[\left(\nabla^{\prime} \otimes \nabla^{\prime}\right) \nabla\right]_{\theta} .
$$

The term $\frac{1}{2} \operatorname{tr}\left[V\left(\hat{\theta}_{d}\right) \underline{h}_{\theta \theta}\right]$ equals $\frac{1}{4} Z^{\prime} E[B] Z$, where

$$
B=2(\operatorname{vec} H)(\operatorname{vec} H)^{\prime}+G \otimes \nabla+\nabla^{\prime} \otimes G^{\prime}+F
$$

and $G$ and $F$ denote the third and fourth derivative matrices. Differentiating $E[H]$ twice yields

$$
E[H]_{\theta \theta}=E[F]+E[G \otimes \nabla]+E\left[\nabla^{\prime} \otimes G^{\prime}\right]+E\left[(\operatorname{vec} H)(\operatorname{vec} H)^{\prime}\right]+E\left[\nabla^{\prime} \otimes H \otimes \nabla\right]
$$

$E[B]$ thus reduces to

$$
E[B]=E\left[(\operatorname{vec} H)(\operatorname{vec} H)^{\prime}-\nabla^{\prime} \otimes H \otimes \nabla\right]+E[H]_{\theta \theta} .
$$

Subtracting twice (B.5) from (B.3) yields (4.21). Parts b and c are immediate consequences of (4.21).

Q.E.D.

\footnotetext{
${ }^{9}$ There are several possible arrangements of higher order derivatives. We are working with arrangements that are compatible with a square fourth derivative matrix.
} 


\section{REFERENCES}

BAIllie, R. T. (1980): "Predictions from ARMAX Models," Journal of Econometrics, 12, 365-374. (1981): "Prediction from the Dynamic Simultaneous Equation Model with Vector Autoregressive Errors," Econometrica, 49, 1331-1337.

Bianchi, C., AND G. Calzolari (1980): "The One-Period Forecast Errors in Nonlinear Econometric Models," International Economic Review, 21, 201-208.

Brown, B., AND R. MARIano (1985): "Asymptotic Behavior of Predictors in Dynamic Nonlinear Simultaneous Systems," Mimeo.

(1984): "Residual-Based Procedures for Prediction and Estimation of a Nonlinear Simultaneous System," Econometrica, 52, 321-343.

Butler, R. W. (1986): "Predictive Likelihood Inference with Applications," Journal of the Royal Statistical Society, Series B, 48, 1-38.

Cooley, T. F., AND W. R. Parke (1987): "Likelihood and Other Approaches to Prediction in Dynamic Models," Journal of Econometrics, 35, 119-142.

Cooley, T. F., W. R. PARKe, and S. Chib (1989): "Predictive Efficiency for Simple Non-Linear Models," Journal of Econometrics, 40, 33-44.

CoX, D. R., AND D. V. Hinkley (1974): Theoretical Statistics. London: Chapman and Hall.

ENGLE, R. F. (1982): "Autoregressive Conditional Heteroskedasticity with Estimates of the Variance of United Kingdom Inflation," Econometrica, 50, 987-1007.

FAIR, R. C. (1980): "Estimating the Expected Predictive Accuracy of Econometric Models," International Economic Review, 21, 355-378.

Hinkley, D. (1979): "Predictive Likelihood," The Annals of Statistics, 7, 718-728.

Kullback, S. (1959): Information Theory and Statistics. New York: John Wiley and Sons.

LAuritzen, S. L. (1974): "Sufficiency, Prediction and Extreme Models," Scandinavian Journal of Statistics, 1, 128-134.

Mariano, R., AND B. Brown (1983): “Asymptotic Behavior of Predictors in a Nonlinear Simultaneous System," International Economic Review, 21, 523-536.

Muench, T., A. Rolnick, N. Wallace, and W. Weiler (1974): "Tests for Structural Change and Prediction Intervals for the Reduced Forms of Two Structural Models of the U.S.," Annals of Economic and Social Measurement, 3/3, 491-519.

Neudecker, H. (1969): "Some Theorems on Matrix Differentiation with Special Reference to Kronecker Matrix Products," Journal of the American Statistical Association, 64, 953-963.

Pfanzagl, J. (1973): “Asymptotic Expansions Related to Minimum Contrast Estimators,” Annals of Statistics, 1, 993-1026.

Phillips, P. C. B. (1979): “The Sampling Distribution of Forecasts from a First-Order Autoregression," Journal of Econometrics, 9, 241-261.

RAO, C. R. (1963): “Criteria of Estimation in Large Samples,” Sankhyā, 25, 189-206. 